\title{
Automatic Detection of Nodules Attached to Vessels in Lung CT by Volume Projection Analysis
}

\author{
Guo-Qing Wei, Li Fan, and JianZhong Qian \\ Intelligent Vision and Reasoning Department \\ Siemens Corporate Research, Inc. \\ 755 College Road East, Princeton NJ 08536, USA
}

\begin{abstract}
Automatic detection of abnormalities or lesions that are attached to other anatomies in medical image analysis is always a very challenge problem, especially when the lesions are small. In this paper a novel method for the automatic detection of lung cancers or nodules attached to vessels in high-resolution multi-slice CT images is presented. We propose to use volume projection analysis to mimic physicians' practices in making diagnosis. The volume projection analysis is performed on 1-dimensional curves obtained from the 3-dimensional volume. A multiscale detection framework is proposed to detect nodules of various sizes. A set of features for characterizing nodules is defined. Results of experimental evaluation of the method are presented.
\end{abstract}

\section{Introduction}

The use of low dose CT to detect early stage of lung cancer as a screening method has shown great promises [2]. The early detection and treatment of lung cancer is vitally important in improving the cure rate. However, the number of $\mathrm{CT}$ slices per patient produced by multi-detector CT systems is usually in the range of 300 to 600 or even more, and the number of screenings made yearly is increasing dramatically [3]. This high volume of patient data virtually makes the softcopy reading slice-by-slice very difficult or impossible in clinical practice. To mitigate the problem, a computer system, which can automatically detect nodules and provide assistance to physicians in the process of diagnostic decision making, is highly desirable.

Early work on computer aided nodule detection relied on 2D features in multiple slices to distinguish nodules from vessels. Giger et al. [8] used multilevel thresholding to binarize individual slices to extract features, such as compactness, circularity, and perimeters. A tree structure relating segmentations in neighboring slices is formed to analyze the $3 \mathrm{D}$ shape of the suspected region. Recent work on nodule detection has focused on the use of 3D features directly to perform the detection. Armato et al applied both intensity and 3D morphological features to classify nodules and non-nodules [4,5]. Kawata et al [6] employed $3 \mathrm{D}$ curvature, texture and moment features to characterize begin and malignant nodules. A major problem with existing nodule detection methods lies in the high false positive rate. With a sensitivity of around $80 \%$, typical false positive

T. Dohi and R. Kikinis (Eds.): MICCAI 2002, LNCS 2488, pp. 746-752 2002.

(C) Springer-Verlag Berlin Heidelberg 2002 
(FP) rate is in the range 1 to 3 per slice [5,1]. This amounts to a few hundred FPs per case for a 200-slice volume, which is too high to be acceptable in a clinical practice. Better results were achieved recently by using patient-specific models in follow-up, with a specificity of 11 FPs per case [9]. Although it is difficult to make a consistent comparison of the different methods because of the different data set used, the above numbers do reflect the current state of the art in CT lung nodule detection.

One of the reasons why current algorithms generate too many false positives is because of the similarities of the extracted features between nodules that are attached to vessels and vessels. For example, nodules attached to vessels have many common characteristics with vessel bifurcations to a computer. Nodules attached to vessels or with vessel-feeding patterns, however, are more likely to be lung cancers and have higher clinical significance. The detection of such nodules is thus very important. The usual way of handling nodules attached to vessels is to use morphological operations, such as opening, to detach nodules from vessels $[3,4]$. Vessel bifurcations, however, when repeatedly opened, may exhibit similar shapes as nodules, and thus cause false detection. Methods that do not try to detach nodules from vessels, but use some statistical measurement of the volume of interest $[5,6]$ suffer from another problem: contributions from the vessel part cannot be separated well from those from the nodule. One has to lower the acceptance threshold in order to increase sensitivity, admitting also, at the same time, more non-nodule anatomies.

In this paper, we propose a novel approach to the detection of nodules attached to vessels. The method mimics physicians' practices in detection of nodules from CT studies in soft-copy reading process. When physicians are examining the CT slices, they often view the axial slices in alternating forward and backward directions along the body-long-axis, and use size change information and patterns of the object of interest to make judgment. We propose to use volume projection analysis to extract the same information the physicians are relying on. The volume projection analysis is based on the analysis of several 1-dimensional curves that are obtained as the projection of the volume from preferred directions. The preferred directions are automatically computed by eigen-value analysis of the volume of interest. To do quantitative shape analysis of the projection curves, Gaussian curve fitting is first conducted on the projection data. Then classification is made in the parameter space of the fitted curves.

\section{Volume Projection Analysis (VPA) Method}

\subsection{Nodule Model}

Most previous methods either implicitly or explicitly use a sphere as the nodule model. For example, the widely accepted spheracity measurement is based on the assumption of a spherical nodule shape, whereas other methods explicitly search for nodules by spherical templates [1]. In this paper an ellipsoidal model is proposed. The reason why ellipsoidal model was not adopted before is probably because of the complexity of the model in comparison with a spherical one. 
Six parameters are involved in an ellipsoidal model instead of one for a spherical model. They are the lengths along the three major axes and the three orientation parameters of the ellipsoid. Without loss of generality, we express the ellipsoid in its own coordinate system (the nodule coordinate system) by

$$
\frac{x^{2}}{a^{2}}+\frac{y^{2}}{b^{2}}+\frac{z^{2}}{c^{2}}=1
$$

with the x,y,z axes coincident with the major axes, and $a, b, c$ are the lengths of the major axes. The orientation of the nodule coordinate system with respect to the original volume coordinate system defines the orientation of the ellipsoid.

It is observed that solitary nodules tend to follow a Gaussian intensity distribution. The intensity has the highest value at the center of the nodule and drops off exponentially in the radial directions. For the ellipsoidal nodule model, the intensity profile can be approximated as

$$
I(x, y, z)=\rho e^{-\left(\frac{x^{2}}{\sigma_{c}^{2}}+\frac{y^{2}}{\sigma_{b}^{2}}+\frac{z^{2}}{\sigma_{c}^{2}}\right)}
$$

where the Gaussian sizes $\sigma_{a}, \sigma_{b}, \sigma_{c}$ are linearly proportional to the lengths of the major axes of the ellipsoid.

\subsection{Multiscale Smoothing}

Due to image noises, it is necessary to smooth the volume. Furthermore, malignant nodules are often more irregular in shape than benign ones; star-like shapes are often to be seen for such nodules. Smoothing will help to reduce such shape irregularity. We use Gaussians of multiple scales for the smoothing

$$
G_{\sigma}(x, y, z)=e^{-\frac{x^{2}+y^{2}+z^{2}}{\sigma^{2}}}
$$

The smoothed volume is represented by

$$
I_{\sigma}(x, y, z)=I(x, y, z) * G_{\sigma}(x, y, z)
$$

where ${ }^{*}$ represents convolution. It can be shown that the smoothed intensity distribution of a Gaussian model is still a Gaussian, with the new Gaussian sizes being

$$
\sigma_{x}=\sqrt{\sigma^{2}+\sigma_{a}^{2}}, \quad \sigma_{y}=\sqrt{\sigma^{2}+\sigma_{b}^{2}}, \quad \sigma_{z}=\sqrt{\sigma^{2}+\sigma_{c}^{2}}
$$

\subsection{Volume Projection}

Volume projection is an operation that reduces the 3-dimensional (3D) data of the volume to a $1 \mathrm{D}$ curve. Given a projection direction vector $v$, the projection axis $\zeta$ is defined in the same direction as $v$, with the origin the same as the original volume of interest. Denote the coordinate on the projection axis by $\zeta$, then the projection of volume $I_{\sigma}(x, y, z)$ on $\zeta$ can be expressed as

$$
p_{v}(\zeta)=\sum_{(x, y, z) \in x \mid x \bullet v=\zeta} I_{\sigma}(x, y, z)
$$

where $\mathbf{x}=(x, y, z)$. The meaning of $(6)$ is to compute the total voxel intensity on the plane orthogonal to $v$ and at a distance $\zeta$ from the origin. The projection 
operation possesses several nice features. The following two are essential in our nodule detection algorithm.

Property 1 (Invariance): The projection of a Gaussian-distributed volume $I_{\sigma}$ $(x, y, z)$ along any projection direction $v$ is still a Gaussian.

Property 2 (Boundness): The size of the projected Gaussian is bounded by

$$
\min \left(\sigma_{x}, \sigma_{y}, \sigma_{z}\right)<\sigma_{\zeta}<\max \left(\sigma_{x}, \sigma_{y}, \sigma_{z}\right)
$$

The first property means that the Guassian shape is invariant under projection operation. This allows us to use lower dimensional data to identify the shape of a higher dimensional volume. Property two, when combined with equation (5), states that the measured shape in the lower dimensional space is quantitatively related to that in the higher dimensional space. Therefore it is feasible to use the projection data to infer the shape of the original volume. Note that by applying the volume projection, we have avoided doing the actual fitting in the original $3 \mathrm{D}$ space, which would be otherwise needed to extract the nodule shape and size information. When the projection axes are chosen as the three major axes of the ellipsoid, it is even possible to approximately reconstruct the $3 \mathrm{D}$ structure from the $1 \mathrm{D}$ measurements.

\subsection{Computing the Projection Axes}

The projection axes should be selected to best distinguish nodules from nonnodule structures. For nodules, the invariance and boundness properties should result in consistent measurements in any projection axes, whereas for non-nodule anatomies, such as vessels, measurements in the projection data will give inconsistent predictions about the 3D shape a nodule structure would exhibit. For example, when one projection axis is chosen as being along the vessel axis, and the other being orthogonal to the vessel axis, the two projection curves will not match any projection curves generated by a nodule. We compute the projection axes based on the eigenvector analysis of the volume of interest. The projection axes are selected corresponding to the dominant structures in the volume of interest.

\subsection{Classification}

To make quantitative analysis of the projected data, Gaussian fitting is conducted for each of the projected curves. Since the data is $1 \mathrm{D}$, the fitting is much simplified in comparison with that in 3D. A Gaussian curve will take the following form

$$
G(\zeta)=\rho_{\zeta} e^{-\frac{\left(\zeta-\zeta_{0}\right)^{2}}{\sigma_{\zeta}^{2}}}
$$

where $\rho_{\zeta}, \zeta_{0}$ and $\sigma_{\zeta}^{2}$ are the Gaussian parameters. A five dimensional feature vector is extracted from all the fittings. It consists of the maximum size, minimum size, maximum size ratio, maximum center offset, and maximum error of the fitting. A simple linear classifier is then used to make distinction between nodules and non-nodule structures. 
After a nodule is detected, the center of the nodule in 3D can be estimated from the Gaussians' centers in 1D by the least squares method. Although the exact size of the nodule is difficult to estimate, we can compute a mean radius of the nodule by averaging the size estimates from the 1D projections. Alternatively, we can use ellipsoidal surface fitting to get more accurate shape information. Note that this is different from a fitting intended for nodule detection, since the volume of interest has already been identified as containing a nodule.

\section{Experiments}

\subsection{Materials}

We applied the proposed method to 10 CT screening studies of smokers. The data sets are all low dose multi-slice, high resolution CT images, with dosages from 20 to $40 \mathrm{mAs}$, in-plane resolutions from 0.57 to $0.67 \mathrm{~mm} /$ pixel, slice thickness of $1.25 \mathrm{~mm}$ with $0.25 \mathrm{~mm}$ overlap. The image sizes are all $512^{*} 512$ within cross section, with 280 to 300 slices in $\mathrm{Z}$ direction.

\subsection{Preprocessing}

First, the lung volume is pre-processed to remove the chest wall so that only the lung area remains as the volume of interest. This is performed by intensity thresholding and morphological operations.

\subsection{Seed Points Generation}

This step generates points of interest in the volume to examine. These points could be specified by scanning through the whole CT volume. To do this, only points whose intensities are greater than a certain threshold need to be considered. However, this is a rather time-consuming approach since the number of candidate points thus generated is huge. We use an intelligent seed point generation method [10] to speed up the detection.

If the nodule detection method is intended to work in an interactive way, the user can pick up seed points manually, e.g., by using a computer mouse to move to the suspicious point and to detect nodules on-line.

\subsection{Ground Truth}

Three chest radiologists first evaluated the patient studies on $7 \mathrm{~mm}$ hard copies separately. Meanwhile, the proposed automatic detection is applied to thin slice multi-slice HR CT data. Finally, two experienced chest radiologists examined all the marks detected by both radiologists and computer, and determined ground truth by consensus.

\subsection{Results}

Preliminary results show that radiologists plus the automatic detection algorithm detected 34 nodules in total, two of which are ground glass nodules (GGNs) detected by radiologists. The radiologists combined together detected 25 nodules. Individual detection ranges from 14 to 21, with the sensitivities ranging 
Table 1. Experimental results of the proposed lung nodule detection method.

\begin{tabular}{|l|c|c|c|c|c|}
\hline \multicolumn{7}{|c|}{ All nodules confirmed: 34 } \\
\hline & $\begin{array}{c}\text { Nodules } \\
\text { detected }\end{array}$ & Sensitivity \% & $\begin{array}{c}\text { Sensitivity } \\
\text { with VPA *\% }\end{array}$ & $\begin{array}{c}\text { Overlap } \\
\text { with computer }\end{array}$ & Overlap rate \% \\
\hline Radiologist 1 & 21 & 61.8 & 97.1 & 11 & 52.4 \\
\hline Radiologist 2 & 17 & 50.0 & 85.3 & 11 & 64.7 \\
\hline Radiologist 3 & 14 & 41.2 & 88.2 & 7 & 50.0 \\
\hline Automatic detection & 23 & 67.6 & - & FPs/study: 6.2 \\
\hline \multicolumn{7}{|c|}{ All nodules confirmed: 32 } \\
\hline \multicolumn{7}{|c|}{$\begin{array}{l}\text { Nodules } \\
\text { detected }\end{array}$} & Sensitivity \% & $\begin{array}{c}\text { Sensitivity } \\
\text { with VPA * \% }\end{array}$ & $\begin{array}{c}\text { Overlap } \\
\text { with computer }\end{array}$ & Overlap rate \% \\
\hline Radiologist 1 & 19 & 59.4 & 96.9 & 11 & 57.9 \\
\hline Radiologist 2 & 16 & 50.0 & 87.5 & 11 & 68.8 \\
\hline Radiologist 3 & 12 & 37.5 & 87.5 & 7 & 58.3 \\
\hline Automatic detection & 23 & 71.9 & - & FPs/study: 6.2 \\
\hline
\end{tabular}

*: Sensitivity with VPA refers to the sensitivities that radiologists can achieve with the help of the automatic nodule detection algorithm.

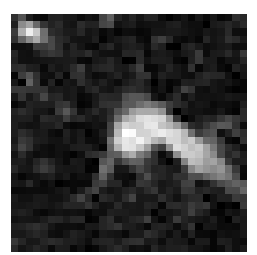

(a)

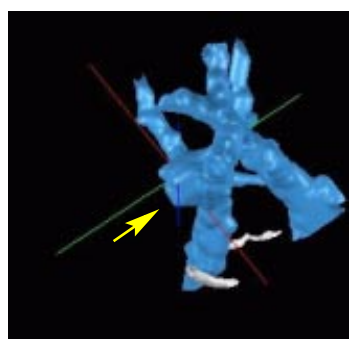

(b)

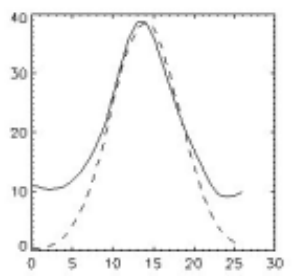

(c)

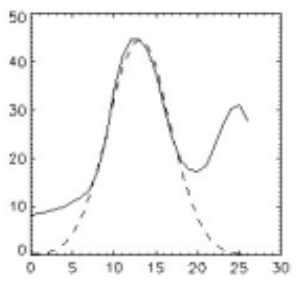

(d)

Fig. 1. Volume projection profiles and curve fitting for a nodule attached to vessel. (a) one slice of theVOI; (b) a shaded surface views of the VOI; the nodule position is indicated by the arrows; (c)-(d) the volume projection curves (solid lines) and fitted curves (dashed lines).

from $41.2 \%$ to $61.8 \%$. The proposed automatic detection algorithm detected 23 nodules, achieving a sensitivity of $67.6 \%$ with a false positive rate of $6.2 \mathrm{FPs}$ per case. The overlapping rate between the automatic detection and each individual radiologist's detection ranges from $50.0 \%$ to $64.7 \%$. With the assistant of automatic detection, radiologists can detect an average of $80.7 \%$ more nodules than alone, and reach the sensitivities ranging from $88.2 \%$ to $97.1 \%$. If excluding the GGNs, the radiologists combined together detected 23 nodules. Individual detection ranges from 12 to 19, with the sensitivities ranging from $37.5 \%$ to $59.4 \%$. The proposed automatic detection algorithm detected 23 nodules, with a sensitivity of $71.9 \%$. The overlapping rate between the automatic detection and each individual radiologist's detection ranges from $58.3 \%$ to $68.8 \%$. With the assistant of automatic detection, radiologists can detect an average of $90.5 \%$ more nodules than alone, and reach the sensitivities ranging from $87.5 \%$ to $96.9 \%$. The overall experimental results are listed in Table 1.

An example of the detection using volume projection data is shown in Fig.1. 


\section{Conclusions}

In this paper, we have presented a novel detection method based on volume projection analysis for solving the problem of automatic detection of lesions that are attached to other anatomies in medical image analysis, especially when the lesions are small. The method projects the $3 \mathrm{D}$ volume data onto $1 \mathrm{D}$ space and extracts the 3D information from the reduced data space. An ellipsoidal nodule model is used to represent a nodule. Under the Gaussian intensity model, two fundamental properties of the projection are established and utilized to design the nodule detection algorithm. The method is able to detect both isolated nodules and nodules attached to vessels.

The method has been applied to 10 patient studies. A low false positive rate of 6.2 FPs per study is achieved, with a comparable sensitivity to existing methods. Experiments have shown that with the help of the proposed method, physicians can detect significantly more nodules than working without.

\section{References}

1. Y. Lee, T. Hara, H. Fujita, S. Itoh, and T. Ishigaki, "Automated detection of pulmonary nodules in helical CT images based on improved template-matching technique", IEEE Trans Medical Imaging, Vol,20, No.7, 2001, pp.595-604

2. C. I Henschke, D. I. McCauley, D. F. Yankelevitz, D. P. Naidich, G. McGuinness, O. S. Miettinen, D. M. Libby, M.W. Pasmantier, J. Koizumi, N. K. Altorki, J. P. Smith, "Early lung cancer action project: overall design and findings from baseline screening," The Lancet, Vol.354, July 10, 1999, pp.99-105

3. A.P. Reeves, W. J. Kostis, "Computer-aided diagnosis of small pulmonary nodules," Seminars in Ultrasound, CT, and MRI, Vol.20, No.2, 2000, pp.116-128

4. S.G. Armato III, M.L. Giger, J.T. Blackburn, K. Doi, H. MacMahon, "Threedimensional approach to lung nodule detection in helical CT", Proc. SPIE Medical Imaging, pp.553-559, 1999

5. S.G. Armato III, M.L. Giger, K. Doi, U. Bick, H. MacMahon, "Computerized lung nodule detection: comparison of performance for low-dose and standard-dose helical Ct scans", Proc. SPIE Medical Imaging, pp.1449-1454, 2001

6. Y. Kawata, N. Niki, H. Ohmatsu, M. Kusumotot, R. Kakinuma, K. Mori, H. Nishiyama, K. Eguchi, M. Kaneko, N. Moriyama, "Curvature based characterization of shape and internal intensity structure for classification of pulmonary nodules using thin-section CT images", Proc. SPIE Medical Imaging, pp.541-552, 1999

7. J. Qian, L. Fan, G.-Q. Wei, C.L. Novak, B. Odry, H. Shen, L. Zhang, D.P. Naidich, J.P. Ko, A.N. Rubinowitz, G. McGuiness, G. Kohl, E. Klotz, "Knowledge-based automatic detection of multi-type lung nodules from multi-detector CT studies", Proc. SPIE Medical Imaging, 2002, to appear

8. M.L. Giger, K.T. Bae, H. MacMahon, "Computerizied detection of pulmonary nodules in computed tomography images", Investigat. Radiol., Vol.29, pp.459-465, 1994

9. M.S. Brown, M.F. McNitt-Gray, J. G. Goldin, R.D. Suh, J.W. Sayre, D.R. Aberle, "Patient-specific models for lung nodule detectionand surveillance in CT images," IEEE Trans Medical Imaging, Vol.20, No.12, 2001, pp.1242-1250

10. L. Fan, J. Qian, G.Q. Wei, "Automatic Generation of Vessel-feeding Pulmonary Nodule Candidates from High-resolution Thin-slice CT Data", US Patent, pending, 2001 